\title{
ACKNOWLEDGMENT OF REVIEWERS
}

The success of Molecular Plant-Microbe Interactions depends on the quality of manuscripts submitted by authors and on the care and competence with which they are reviewed. It is the policy of the Editorial Board to solicit reviews of manuscripts from specialists most qualified to review them. In addition to members of the Editorial Board, the individuals listed below provided constructive critical reviews of one or more manuscripts during the past year. Their names are published here in grateful appreciation for their contributions to the journal.

P. Abad, I.N.R.A., Antibes Cedex, France

J. R. Alfano, University of Nevada, Las Vegas, NV, U.S.A.

C. Allen, University of Wisconsin, Madison, WI, U.S.A.

R. Amasino, University of Wisconsin, Madison, WI, U.S.A.

A. Anderson, Utah State University, Logan, UT, U.S.A.

C. W. Bacon, University of Georgia, Athens, GA, U.S.A.

M. J. Bailey, Institute of Virology and Environmental Microbiology, Oxford, U.K.

S. J. Barker, University of Western Australia, Nedlands, Australia

F. Banuett, University of California, San Francisco, CA, U.S.A.

H. Barker, Scottish Crop Research Institute, Invergowrie, Dundee, Scotland, U.K.

M.-A. Barny, INRA, Paris, FRANCE

J. Batut, UNR215 CNRS INRA, Castanet Tolosan, France

T. J. Baum, Iowa State University, Ames, IA, U.S.A.

G. Beattie, Iowa State University, Ames, IA, U.S.A.

G. Becard, CNRS Universite Paul Sabatier, Castanet Tolosan, France

A. Becker, University Bielefeld, Bielefeld, Germany

E. J. Bedmar, Estacion Experimental del Zaidin, CSIC, Granda, Spain

S. Beer, Cornell University, Ithaca, New York, U.S.A.

A. Bent, University of Wisconsin, Madison, WI, U.S.A.

D. Bergey, Montana State University, Bozeman, MT, U.S.A.

D. M. Bisaro, Ohio State University, Columbus, OH, U.S.A.

T. Bisseling, Wageningen University, Wageningen, The Netherlands

E. Blancaflor, Samuel Roberts Noble Foundation, Ardmore, OK, U.S.A.

A. J. Bogdanove, Iowa State University, Ames, IA, U.S.A.

J. Bol, Leiden University, Leiden, The Netherlands

U. Bonas, Martin-Luther University, Halle (Saale) Germany

P. Bonfante, University of Torino, Torino, Italy

K. Boot, Leiden University, Leiden, The Netherlands

C. Boucher, INRA-CNRS, Castinet Tolosan, France

W. J. Broughton, Universite de Geneve, Geneve, Switzerland

V. Buchanan-Wollaston, Horticulture Research International, Wellesbourne, Warwick, U.K.

W. Bushnell, University of Minnesota, St. Paul, MN, U.S.A.

M. X. Caddick, University of Liverpool, Liverpool, U.K.

R. Carlson, University of Georgia, Athens, GA, U.S.A.

J. Carrington, Washington State University, Pullman, WA, U.S.A.

C. E. Caten, University of Birmingham, Birmingham, U.K.

C. J. Chang, University of Georgia, Griffin, GA, U.S.A.

L. M. Ciuffetti, Oregon State University, Corvallis, OR, U.S.A.

D. B. Collinge, Royal Veterinary and Agricultural University, Frederiksberg, Denmark
A. Collmer, Cornell University, Ithaca, NY, U.S.A

I. Connerton, University of Nottingham, Loughborough, U.K.

D. Cook, University of California, Davis, CA, U.S.A.

F. Cote, University of Georgia, Athens, GA, U.S.A.

S. Covert, University of Georgia, Athens, GA, U.S.A.

S. Covey, John Innes Centre, Norwich, U.K.

M. Crespi, CNRS, Gif sur Yvette, France

L. Crossman, Sainsbury Laboratory, Norwich, U.K.

J. Culver, University of Maryland, College Park, MD, U.S.A.

R. A. Dean, Clemson University, Clemson, SC, U.S.A.

F. Debelle, INRA-CNRS, Castanet-Tolosan, France

T. Denny, University of Georgia, Athens, Georgia, U.S.A.

Y. Desseaux, ISV-CNRS, Gif-sur-Yvette, France

C. Diaz, Leiden University, Leiden, The Netherlands

M. Dickman, University of Nebraska, Lincoln, NE, U.S.A.

M. A. Djordjevic, Australian National University, Canberra City, Australia

J. Doonan, John Innes Centre, Norwich, U.K.

J. M. Dow, Sainsbury Laboratory, Norwich, U.K.

D. Dowling, Institute of Technology Carlow, Carlow, Ireland

J. A. Downie, John Innes Centre, Norwich, U.K.

M. Dufresne, Centre de Recherche sur les Plantes, Orsay, France

D. Dunigan, University of Florida, Dover, FL, U.S.A.

J. Duvick, Pioneer Hi-Bred, Johnston, IA, U.S.A.

J. Ellis, CSIRO, Division of Plant Industry, Canberra, Australia

C. Elmerich, Institut Pasteur, Paris, France

P. Epple, University of North Carolina, Chapel Hill, NC, U.S.A.

M. Estelle, The University of Texas at Austin, Austin, TX, U.S.A.

T. Eulgem, University of North Carolina, Chappel Hill, NC, U.S.A.

M. L. Farman, University of Kentucky, Lexington, KY, U.S.A.

T. Finan, McMaster University, Hamilton, Ontario, Canada

J. Fletcher, Oklahoma State University, Stillwater, OK, U.S.A.

P. Franken, Max-Planck-Institut fur Terrestriche Mikrobiologie, Marburg, Germany

C. Fuqua, Indiana University, Bloomington, IN, U.S.A.

T. Gaffney, Novartis Agribusiness Biotechnology Research, Research Triangle Park, NC, U.S.A.

K. Geider, Max-Planck-Institut Zellbiologie, Ladenburg, Germany

D. M. Geiser, Pennsylvania State University, University Park, PA, U.S.A.

S. Gelvin, Purdue University, West Lafayette, IN, U.S.A.

J. Gershenzon, Max Planck Institute of Chemical Ecology, Jena, Germany

D. Gilchrist, University of California-Davis, Davis, CA, U.S.A.

R. Goldbach, Wageningen Agricultural University, Wageningen, The Netherlands 
S. Gold, University of Georgia, Athens, GA, U.S.A.

M. I. Gonzales Roncero, Universidad de Córdoba, Cordoba, Spain

C. Gonzalez, Texas A\&M University, College Station, TX, U.S.A.

N. Goosen de Roo, Leiden University, Leiden, The Netherlands

K. Goulter, John Hines Building, Brisbane St. Lucia, Australia

A. Goverse, Wageningen University, Wageningen, The Netherlands

J. Green, University of Birmingham, Birmingham, U.K.

N. Grimsley, CNRS-INRA, Castanet-Tolosan, France

S. Gurr, University of Oxford, Oxford, U.K.

C. Gutierriez, Universidad Autonoma de Madrid, Madrid, Spain

D. Haas, Universite de Lausanne, Lausanne-Dorigney, Switzerland

M. Hahn, Universitaet Konstanz, Konstanz, Germany

J. Hamer, Paradigm Genetics Ins. 104, Research Triangle Park, NC, U.S.A.

K. Hammond-Kosack, Monsanto plc, Trumpington, Cambridge, U.K.

J. Handelsman, University of Wisconsin-Madison, Madison, WI, U.S.A.

A. Hardman, University of Nottingham, Nottingham, U.K.

J. Hargreaves, IACR-Long Ashton Research Station, Long Ashton, Bristol, U.K.

S. Y. He, Michigan State University E. Lansing, MI, U.S.A.

M. Heath, University of Toronto. Toronto, Ontario, Canada

H. Helder, Wageningen University, Wageningen, The Netherlands

J. Hinton, Norwich Research Park, Norwich, U.K.

S. Hirano, University of Wisconsin, Madison, WI, U.S.A.

E. Holub, Horticulture Research International, Warwickshire, U.K.

P. J. J. Hooykaas, Leiden University, Leiden, The Netherlands

Gregg A. Howe, Michigan State University, East Lansing, MI, U.S.A.

Barbara J. Howlett, University of Melbourne, Victoria, Australia

B. Hyman, University of California, Riverside, CA, U.S.A.

N. Iacobellis, Universita degli Studia del Basilicata, Potenzia, Italy

E. Johansen, Danish Institute of Agricultural Sciences, Frederiksberg, Denmark

K. Johnstone, University of Cambridge, Cambridge, U.K.

L. Jouanin, INRA, Versailles, France

H. Judelson, University of California, Riverside, CA, U.S.A.

I. Kaloshian, University of California, Riverside, CA, U.S.A.

Y. Kapulnik, ARO, Volcani Center, Bet Dagan, Israel

P. Katinakis, Agricultural University of Athens, Athens, Greece

K. Kazan, University of Queensland Brisbane, Brisbane, Australia

N. Keen, University of California, Riverside, CA, U.S.A.

G. Kema, DLO Research Institute for Plant Protection, Wageningen, The Netherlands

F. Kempken, Ruhr-Universität Bochum, Bochum, Germany

C. Kennedy, University of Arizona, Tucson, AZ, U.S.A.

J. Kijne, Leiden University, Leiden, The Netherlands

G. King, Horticulture Research International, Warwick, U.K.
C. Kistner, John Innes Centre, Norwich, U.K.D. Kleupfel, Clemson University, Clemson, SC, U.S.A.

D. Y. Kobayashi, Rutgers University, New Brunswick, NJ, U.S.A.

E. Kondorosi, CNRS, Gif-Sur-Yvette, France

K. Korth, University of Arkansas, Fayetteville, AR, U.S.A.

E. Lam, Rutgers University, New Brunswick, NJ, U.S.A.

K. Lambert, University of Illinois, Urbana, IL, U.S.A.

M. Laus, Leiden University, Leiden, The Netherlands

K. A. Lawton, Novartis Crop Protection, Inc., Research Triangle Park, NC, U.S.A.

M.-H. Lebrun, UMR 1032 CNRS-RPA, Lyon, France

B. Lippok,Max Planck Institut Zuechtungsforschung, Koeln, Germany

J. Loper, USDA-ARS, Corvallis, OR, U.S.A.

E. J. J. Lugtenberg, Leiden University, Leiden, The Netherlands

D. Luthe, Mississippi State University, Mississippi State, MS, U.S.A.

G. Lyon, Scottish Crop Research Institute. Invergowrie, Dundee, Scotland, U.K.

I. E. Maldonado-Mendoza, CIIDIR-Sinaloa, Sinaloa, Mexico

J. M. Manners, University of Queensland, Brisbane, St. Lucia, Australia

J. M. Mansfield, Wye College (University of London), Wye, Ashford, Kent, U.K.

F. Martin, Inst Natl Recherche Agronomique, Champenoux, France

E. Martinez-Romero, UNAM, Cuernavaca, MOR, Mexico

A. Matthysse, University of North Carolina, Chapel Hill, NC, U.S.A.

M. Mehdy, University of Texas, Austin, TX, U.S.A.

K. Mendgen, Universität Konstanz, Konstanz, Germany

R. W. Michelmore, University of California, Davis, CA, U.S.A.

D. Milbourne, Scottish Crop Research Institute, Invergowrie, Dundee, U.K.

R. E. Mitchell, Horticulture and Food Research Institute, New

Zealand Ltd., Auckland, New Zealand

R. Mithen, John Innes Centre, Norwich, U.K.

J. Morris, University of Nebraska, Lincoln, NE, U.S.A.

M. B. Mudgett, University of California, Berkeley, CA, U.S.A.

P. Mueller, Phillips Universitat, Marburg, Germany

J. F. Murphy, Auburn University, Auburn, AL, U.S.A.

A. M. Murphy, University of Cambridge, Cambridge, U.K.

R. Nelson, Samuel Roberts Noble Foundation, Ardmore, OK, U.S.A.

E. Nester, University of Washington, Seattle, WA, U.S.A.

D. Noel, Marquette University, Milwaukee, WI, U.S.A.

D. Nuss, University of Maryland, College Park, MD, U.S.A.

F. O'Gara, National University of Ireland, Cork, Ireland

Y. Okon, Katholieke University Leuven, Heverlee, Belgium

R. Oliver, Carlsberg Laboratory, Copenhagen Valby, Denmark

C. Opperman, North Carolina State University, Raleigh, NC, U.S.A.

N. L. Paiva, Samuel Roberts Noble Foundation Inc., Ardmore, OK, U.S.A.

T. Palva, University of Helsinki, Helsinki, Finland

M. Parniske, Sainsbury Laboratory, Norwich, U.K.

E. Partiarca, International Institute of Genetics and Biophysics, Napoli, Italy 
S. Patil, University of Hawaii, Honolulu, Hawaii, U.S.A.

K. Pawlowski, University of Gottingen, Gottingen, Germany

G. Payne, North Carolina State University, Raleigh, NC, U.S.A.

A. Pereira, Plant Research International, Wageningen, The Netherlands

T. Petty, North Carolina State University, Raleigh, NC, U.S.A.

C. Pieterse, Utrecht University, Utrecht, The Netherlands

M. Pirhonen, Swedish University of Agricultural Sciences, Uppsala, Sweden

G. M. Preston, University of Oxford, Oxford, U.K.

N. P. Price, SUNY College of Environmental Science and Forestry, Syracuse, NY, U.S.A.

A. Puehler, University of Bielefeld, Bielefeld, Germany

P. Rainey, University of Oxford, Oxford, U.K.

A. L. N. Rao, University of California, Riverside, CA, U.S.A. J. Rathjen, University of California, Davis, Davis, CA, U.S.A. W. Ream, Oregon State University, Corvallis, OR, U.S.A.

K. Richards, CNRS, Strasbourg Cedex, France

D. Robinson, Scottish Crop Research Institute, Invergowrie, Dundee, Scotland, U.K.

P. Rodriguez-Palenzuela, Ciudad Universitaria s/n, Madrid, Spain

B. Rolfe, The Australian National University, Canberra, Australia

M. Romantschuk, University of Helsinki, Helsinki, Finland

T. Romeis, The Sainsbury Laboratory, Norwich, U.K.

D. Romero, UNAM, Morelos, Mexico

C. Ronson, University of Otago, Dunedin, New Zealand

J. E. Ruiz Sainz, University de Sevilla, Sevilla, Spain

T. Ruiz-Argueso, Universidad Politecnica de Madrid, Madrid, Spain

P. Rushton, Max-Planck-Institut fur Zuechtungsforschung, Koeln, Germany

G. Salmond, University of Cambridge, Cambridge, U.K.

G. Sandberg, Swedish University of Agricultural Sciences, Umea, Sweden

R. Sandrock, Arcaris, Inc., Salt Lake City, UT, U.S.A.

S. Santa Cruz, Scottish Crop Research Institute, Invergowrie, Dundee, Scotland, U.K.

C. Sautter, Institute of Plant Sciences ETH, Zurich, Switzerland

W. Schaefer, Botanical Institut, Hamburg, Germany

C. L. Schardl, University of Kentucky, Lexington, KY

W.R.M. Schlaman, Leiden University, Leiden, The Netherlands

M. Schultze, University of York, York, U.K.

J. Scott-Craig, Michigan State University, East Lansing, MI, U.S.A.

R. Sen, University of Helsinki, Helsinki, Finland

K. Shirasu, The Sainsbury Laboratory, Norwich, U.K.

A. Simon, University of Massachusetts, Amherst, MA, U.S.A.

I. E. Somssich, Max-Planck-Institut f. Zuechtungsforschung, Koeln, Germany
C. Staben, University of Kentucky, Lexington, KY, U.S.A.

G. Stacey, University of Tennessee, Knoxville, TN, U.S.A.

J. Stanley, John Innes Institute, Norwich, U.K.

J. M. Stone, Massachusetts General Hospital, Boston, MA, U.S.A.

D. Tagu, INRA, Champenoux, France

N. J. Talbot, University of Exeter, Exeter, U.K.

S. Tang, University of North Carolina, Chapel Hill, NC, U.S.A.

K. Thevissen, Katholieke University Leuven, Heverlee, Belgium

C. Thomas, Sainsbury Laboratory, Norwich, U.K.

B. Thomma, FA Janssens Laboratory of Genetics, Leuven, Belgium

N. Toro, Estacion Exp. Del Zaidin, Granada, Spain

M. Torres, University of North Carolina, Chapel Hill, NC, U.S.A.

E. Triplett, University of Wisconsin, Madison, WI, U.S.A.

T. Tsuge, Nagoya University, Chikusa, Nagoya, Japan

P. Tudzynski, Westfaelische Wilhelms-Universitaet, Muenster, Germany

B. Tudzynski, Westfaelische Wilhelms-Universitaet, Muenster, Germany

B. Tyler, University of California, Davis, CA, U.S.A.

M. Udvardi, Max Planck Institute for Molecular Plant Physiology, Golm, Germany

B. Valent, DuPont Agricultural Products, Wilmington, DE, U.S.A.

J. van de Leyden, Katholieke University Leuven, Heverlee, Belgium

F. van Gijsegem, Universiteit Gent, Gent, Belgium

J.A.L. van Kan, Wageningen University, Wageningen, The Netherlands

C. Vance, University of Minnesota, St. Paul, MN, U.S.A.

B. Verduin, Wageningen University, Wageningen, The Netherlands

O. Vionnet, Sainsbury Laboratory, Norwich, U.K.

L. Walling, University of California, Riverside, CA, U.S.A.

J. Walton, Michigan State University, East Lansing, MI, U.S.A.

P. J. Weisbeek, Utrecht University, Utrecht, The Netherlands

D. M. Weller, Washington State University, Pullman, WA, U.S.A.

D. Werner, Phillips-Universitat Marburg, Marburg, Germany

M. Whalen, San Francisco State University, San Francisco, CA, U.S.A.

F. F. White, Kansas State University, Manhattan, KS, U.S.A.

D. K. Willis, University of Wisconsin, Madison, WI, U.S.A.

W. Wintermantel, USDA-ARS, Salinas, CA, U.S.A.

K. Wobbe, Worcester Polytechnic Institute, Worchester, MA, U.S.A.

J. -R. Xu, Purdue University, West Lafayette, IN, U.S.A. 